\title{
MicroRNA-520f suppresses growth of gastric carcinoma cells by target ATPase family AAA domain-containing protein 2 (ATAD2)
}

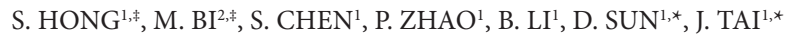 \\ ${ }^{1}$ Department of Colon and Anal Surgery, The first hospital of Jilin University, Jilin University, Changchun, Jilin, China; ${ }^{2}$ Department of Ophthal- \\ mology, The China-Japan Union Hospital of Jilin University, Jilin University, Changchun, Jilin, China \\ *Correspondence: jiandongtai73@163.com, sundy19830000@hotmail.com \\ ${ }^{*}$ Contributed equally to this work.
}

\section{Received January 10, 2016 / Accepted April 25, 2016}

\begin{abstract}
MicroRNAs (miRNAs) are small, non-coding RNAs that can serve as tumor suppressor genes or oncogenes in tumorigenesis. More and more evidence demonstrate that abnormal expression of miRNAs lead to the gastric carcinoma occurrence. In the present study, we revealed that the expression levels of miR-520f were significantly down-regulated in gastric carcinoma cells and clinical gastric carcinoma samples. Next, we demonstrated that introduction of miR-520f inhibited the growth of gastric carcinoma cells in vitro. However, down-regulate the expression levels of miR-520f by anti-miR-520f lead to an enhanced cell proliferation, implying that miR-520f maybe serve as a novel tumor suppressor. Moreover, we found that ATPase family AAA domain-containing protein 2 (ATAD2) was one target genes of miR-520f downstream regulator, which caused the decreased expression of ATAD2. Meanwhile, the overexpression of ATAD2 reversed the inhibited proliferation ability caused by miR-520f. Therefore, our find that miR-520f involves in gastric carcinoma proliferation, pointing a therapeutic probability of miR-520f in the therapy of gastric carcinoma.
\end{abstract}

Keywords: MicroRNA-520f, gastric carcinoma, ATAD2, proliferation

MicroRNAs (miRNAs) are small, non-coding RNA of about 22 nucleotides that negatively regulate gene expression via RNA-induced silencing complex (RISC)-mediated mRNA processing or translational inhibition, when partially complementary sequences are recommend in the 3'untranslated regions (3'UTRs) of the target mRNAs [1]. The biogenesis of miRNAs covers a series of protein system, including the Argonaute family, Pol II-dependent transcription and the RNase IIIs Dicer and Drosha. MiRNAs are genetically standpat and have vital roles in biological processes, including differentiation, development, cell proliferation, immunity, apoptosis and metabolism [2, 3]. Multiple miRNAs are abnormally expressed in human cancers, which were validated by the development of microarray analysis of miRNA expression. At the same time, the molecular characterization of miRNAs connotate that they may serve as a novel class of tumor-suppressor genes or oncogenes [4].

Gastric cancer is developed from the lining of the stomach [5]. Precocious clinical symptoms may involve heartburn, upper abdominal pain, nausea and loss of appetite. Subsequent signs and symptoms may involve weight loss, yellow skin and whites of the eyes, puking, hard swallowing, and blood in the stool in particular. The cancer may extend from the stomach to other proportions of the body, specifically the liver, lungs, bones, lining of the abdomen and lymph nodes. Although in other solid tumors, the development and progression of gastric tumour are linked with additional genetic and epigenetic alterations[6]. However, the cumulative proof so far cannot explain the full theory of gastric carcinoma. Latest studies have emphasized the factitive links between miRNA deregulations and cancer progress [7]. MiRNAs have been found to be abnormally expressed in gastric carcinoma [7].

ATAD2 (ATPase family AAA domain-containing protein 2), a member of the AAA + ATPase family of proteins, contains both a bromodomain and an ATPase domain, and maps to chromosome $8 \mathrm{q} 24$ in a region that is continually expanded in cancer [8]. The structure of ATAD2 proposes that it has functions connected with genome regulation, including cell proliferation, differentiation and apoptosis [9]. Studies have showed that ATAD2 is highly expressed in several sorts of tumors such as breast cancer, lung cancer, and large B-cell lymphoma. It has been reported 
that ATAD2 links the E2F and MYC pathways and contributes to the development of aggressive cancer through the enhancement of MYC-dependent transcription [10]. MiR-372 reduces the oncogene ATAD2 to influence hepatocellular carcinoma proliferation and metastasis [11]. Functional characterization of ATAD2 has been a updated cancer/testis factor and a prophet of poor prognosis in breast and lung cancers [12].

In the current study, we propose to clarify the pathophysiological role of miR-520f in gastric carcinoma tumorigenesis. Our discovery explains that miR-520f is down-regulated in gastric carcinoma cell lines and clinical gastric carcinoma tissues, suggesting that miR-520f might serve as a tumor suppressor. We recognized that ATAD2 is one of the immediate target genes of miR-520f. MiRNA$520 \mathrm{f}$ can restrain the increase of gastric carcinoma cells via aiming at ATAD2.

\section{Materials and methods}

Cell lines and cell culture. HFE145, BGC823, MGC803, SGC7901 and PAMC82 were maintained in Dulbecco's modified Eagle's medium (Gibco, Grand Island, NY). GES-1 lines was grown in RPMI Medium 1640 (Gibco) supplemented with $10 \%$ fetal calf serum (FCS), $100 \mathrm{U} / \mathrm{ml}$ penicillin, and $100 \mathrm{mg} /$ $\mathrm{ml}$ streptomycin in $5 \% \mathrm{CO} 2$ at $37^{\circ} \mathrm{C}$.

RNA extract and qRT-PCR. Total RNA was drawn from cells by Trizol reagent (Invitrogen). First-strand cDNA was compounded as reported before. To analyze miRNA-520f expression, total RNA was polyadenylated by poly (A) polymerase (Ambion, Austin, TX, USA) as described formerly. Reverse transcription was implemented through poly (A)-tailed total RNA and reverse transcription primer with ImPro-II Reverse Transcriptase (Promega, Madison, WI, USA) on the basis of the manufacturer's protocol. QRT-PCR was performed by a Bio-Rad sequence detection system on the basis of the manufacturer's explanations by double-stranded DNA-specific SYBR GreenPremix Ex TaqTM II Kit (TaKaRa Bio, Dalian, China). Experiments were managed in duplicate in three isolated assays. Relative transcriptional folds were computed as $2-\Delta \Delta \mathrm{Ct}$. GAP$\mathrm{DH}$ was applied to an internal control for standardization.

Tissue specimens. Thirty-three gastric carcinoma tissues and their consistent nearby nontumorous liver tissues used in this study were immediately acquired from the first hospital of Jilin University (Jilin, P.R. China) after surgical resection. Clinicopathological knowledge about the patients was acquired from patient register. All patients were diagnosed with early gastric carcinoma, and nobody had received preferred radiotherapy or chemotherapy before the surgery. Written consents approving the use of their tissues for investigation purposes after operation were acquired from each patient. The study agreement was agreed by the Institute Research Ethics Committee.

Oligonucleotides and plasmids. The miR-520f mimic (miR-520f), mimic NC, miR-520f inhibitor (anti-miR-520f) and inhibitor NC (anti-miR-NC) were purchased from RiboBio (Guangzhou, China). The fragment of ATAD2 3'UTR was subcloned into the pGL3 control vector (Promega, Madison, WI, USA), to go on pGL3-ATAD2-3'UTR-wt. Mutant construct of ATAD2-3'UTR, named as pGL3-ATAD2-3'UTRmut, which kept a substitution of four nucleotides within the core seed sequence of miR-520f, was achieved using overlapping spread PCR. The CDS region of ADAT2 was cloned to pcDNA for overexpression in the cells.

Cell transfection. RNA oligonucleotides were transfected through Lipofectamine RNAiMAX (Invitrogen, Carlsbad, CA, USA). RNA transfection efficiency was approximately $70-80 \%$ and the overexpression of miRNA continued for at least 2 days. Lipofectamine 2000 (Invitrogen) was utilized for transfection of plasmid alone or together with RNA oligonucleotides.

Cell proliferation assay. MGC803 or GES- 1 cells were seeded onto 96 well plates ( 2000 cells/well) for $24 \mathrm{~h}$ before transfection and 3-(4,5-dimethylthiazol-2-yl)-2,5-diphenyl-

tetrazolium bromide (MTT) assay was used to measure cell proliferation after transfection. MTT assay was described previously [13]. 5-ethynyl-2'-deoxyuridine (EdU) incorporation assay was processed by the Cell-Light TM EdU imaging detecting kit (RiboBio).

Luciferase reporter gene assay. Luciferase reporter gene assay was performed using the Dual-Luciferase Reporter Assay System (Promega) in accordance with the manufacturer's instructions. Cells were seeded into 24 -well plates at $3 \times 10^{4}$ cells per well. After $24 \mathrm{~h}$, the cells were co-transfected with $0.1 \mu \mathrm{g} /$ well of pRL-TK plasmid (Promega) involved in the Renilla luciferase gene for internal normalization. The luciferase activities were assessed as previously described. All experiments were performed at least three times.

Western blot analysis. Western blot analysis was performed as previously described [15]. The primary antibodies applied to western blot were rabbit polyclonal anti-ATAD2 (Santa Cruz Biotechnol-ogy, Santa Cruz, CA, USA), mouse monoclonal anti-b-actin (Cell Signaling Technology, Beverly, MA, USA).

Statistical analysis. Every experiment was repeated at least three times. Statistical significance was measured by comparing mean values $( \pm \mathrm{SD})$ using a Student's $t$ test for independent groups and was measured for $p<0.05\left(^{*}\right), p<0.01$ $\left({ }^{* *}\right)$ and $\mathrm{p}<0.001\left({ }^{* *}\right)$. MiR-520f expression in tumor tissues and matched adjacent nontumor samples were compared by the Wilcoxon signed rank test.

\section{Results}

Expression levels of miR-520f are decreased in gastric carcinoma cells and gastric carcinoma samples. To confirm this hypothesis, we analyzed the expression of miR-520f in 2 immortalized stomach cell lines, 4 human gastric carcinoma cell lines, 33 paired clinical gastric carcinoma and adjacent nontumorous stomach tissues using quantitative real-time PCR (qRT-PCR) and standardized against an endogenous control (U6 RNA). The results showed that miR-520f was reduced in all the four gastric carcinoma cell lines (Figure 1A). Compared with their nontumorous coun- 
A

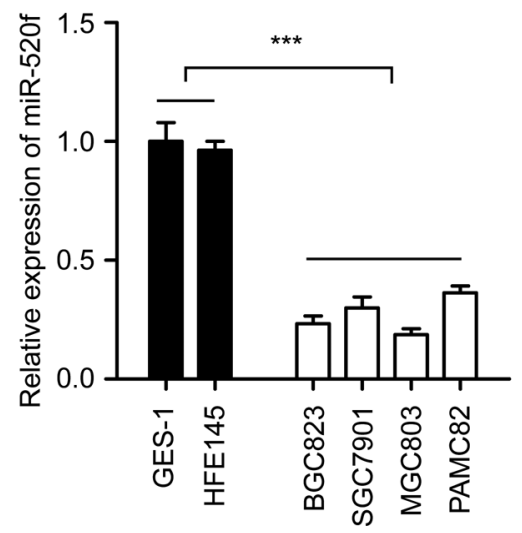

B

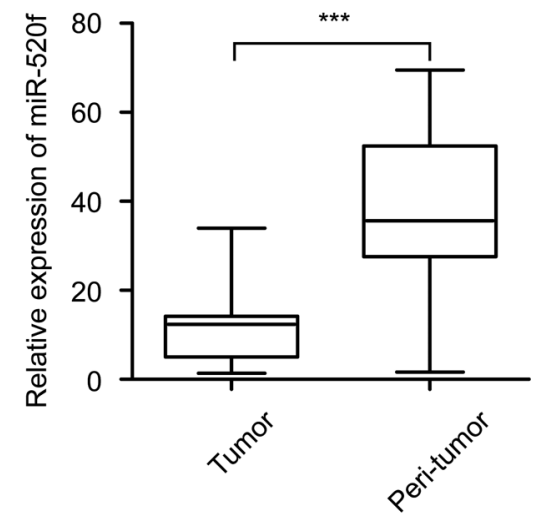

Figure 1. Expression of miR-520f is decreased in gastric carcinoma cells and gastric carcinoma samples.

(A): qRT-PCR analysis of miR-520f expression in normal stomach cells and gastric carcinoma cells and standard by an endogenous control (U6 RNA). The expression of miR-520f was normalized to U6 with respect to GES-1 cells. One-way analysis of variance was used to compare miR-520f expression in all individual normal stomach cells with all gastric carcinoma cell lines. Data are reported as mean \pm s.d. for three independent experiments $(* * \star P<0.001$, Student's t-test). (B): qRT-PCR analysis of miR-520f expression in 33 paired gastric carcinoma tissues and their corresponding nontumorous livers. miR-520f expression in gastric carcinoma tissues and nontumorous liver tissues were compared by way of Wilcoxon signed-rank test $(* \star \star ~ P<0.001$, Student's $t-t e s t)$.

A

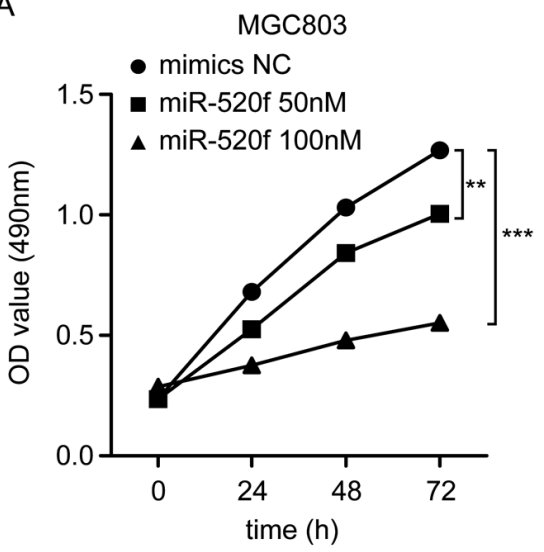

C

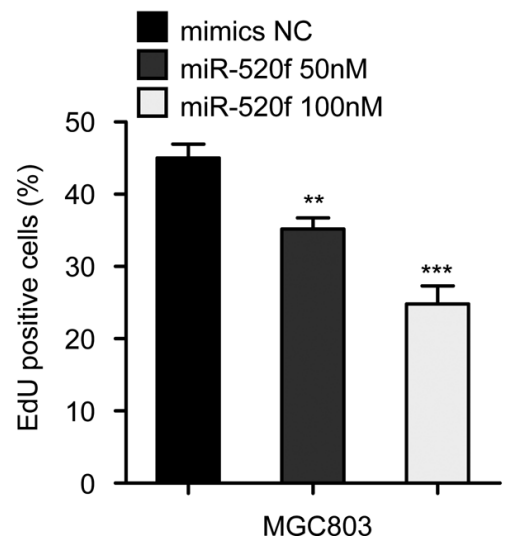

B

GES-1

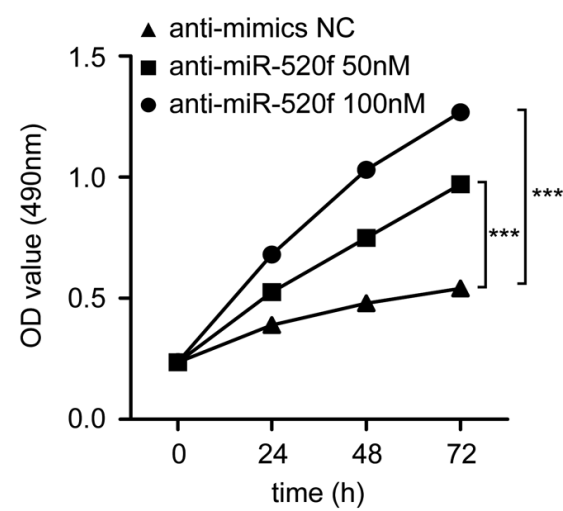

D

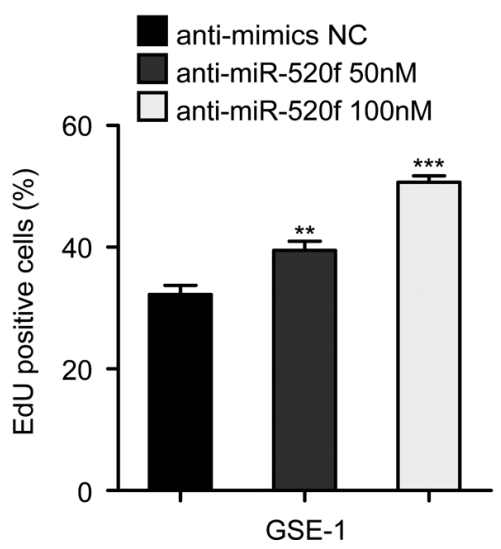

Figure 2. miR-520f inhibits growth of gastric carcinoma cells in vitro.

(A-B): MGC803 cells were transfected with $100 \mathrm{nM} \mathrm{NC}$ and 50 or $100 \mathrm{nM}$ miR-520f, respectively. GES-1 cells were transfected with $100 \mathrm{nM}$ anti-miR$\mathrm{NC}$ and 50 or $100 \mathrm{nM}$ anti-miR-520f, respectively. The effects of miRNAs on cell proliferation were identified by 3-(4,5-dimethylthiazol-2-yl)-2,5diphenyltetrazolium bromide (MTT) assay at 24, 48 and $72 \mathrm{~h}$ after transfection. (C-D): MGC803 cells were transfected with $100 \mathrm{n}$ M NC and $100 \mathrm{nM}$ miR-520f, respectively. GES- 1 cells were transfected with $200 \mathrm{nM}$ anti-miR-NC and $200 \mathrm{nM}$ anti-miR-520f, respectively. The cell proliferation was tested by 5 -ethynyl-2'-deoxyuridine (EdU) incorporation assay at $48 \mathrm{~h}$ after transfection. 
A

ATAD2 3'UTR: 5'-AAUUUUGUUUGUAUUAAGCACUU-3'

||||||||

miR-520f: 3 '-UUGGGAGAUUUUCCUUCGUGAA-5'
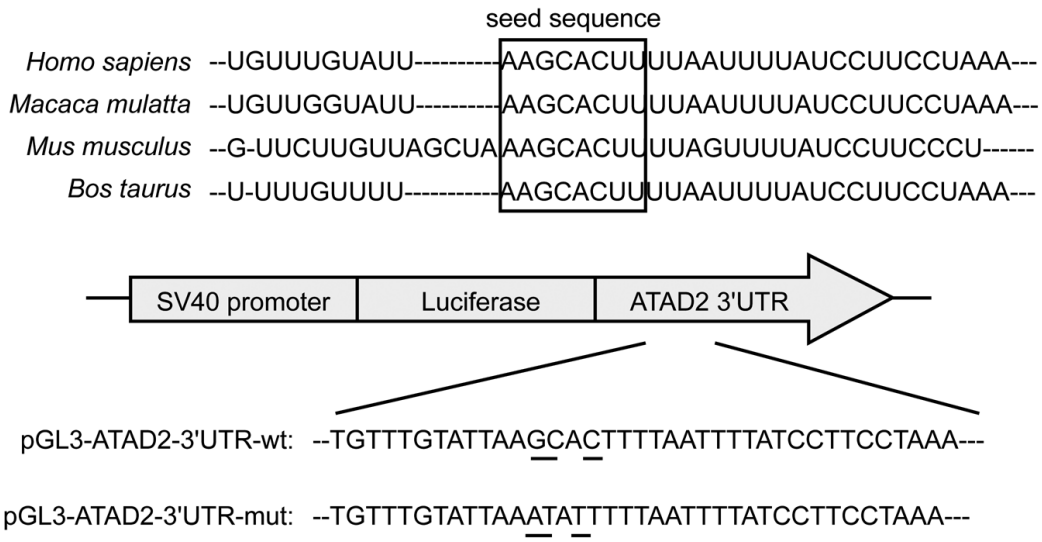

B

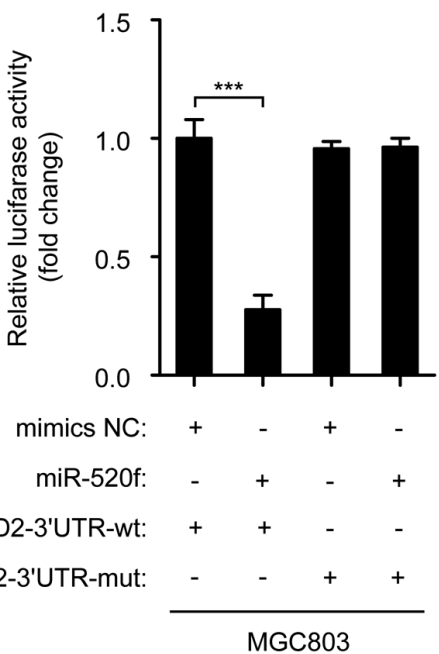

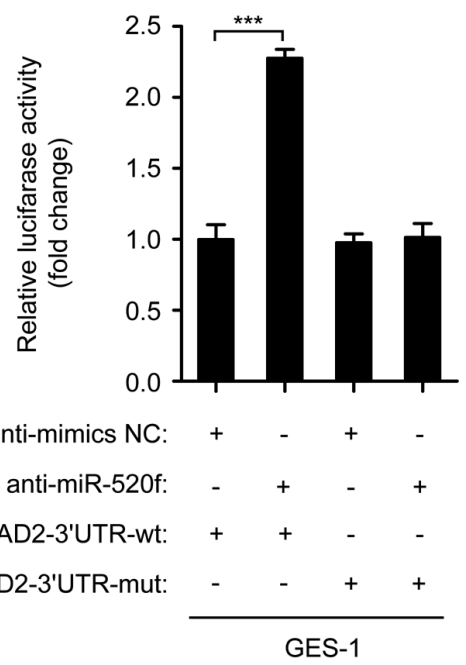

$\mathrm{D}$

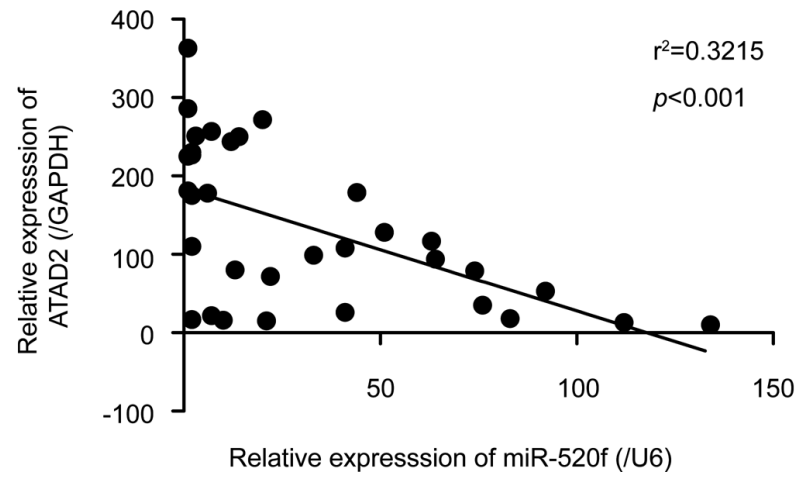

Figure 3. miR-520f directly inhibits the expression of ATAD2 through its 3'UTR.

(A): MiR-520f-binding site is predicted at the 3'UTR of ATAD2 mRNA. Mutant was generated at the ATAD2 3'UTR seed region as indicated. An ATAD2 3'UTR fragment includes wild type or mutant of the miR-520f-binding sequence was cloned into the downstream of the luciferase reporter gene. (B): MGC803 cells were co-transfected with Renilla luciferase plasmid and a firefly luciferase reporter plasmid containing either wild-type or mutant ATAD2 3'UTR (indicated as pGL3- ATAD2-3'UTR-wt and pGL3- ATAD2-3'UTR -mut) with either NC or miR-520f. Luciferase activity was determined $48 \mathrm{~h}$ after transfection. (C): GES-1 cells were cotransfected with Renilla luciferase plasmid and a firefly luciferase reporter plasmid containing either wild-type or mutant ATAD2 3'UTR with either anti-miR-NC or anti-miR-520f. Luciferase activity was determined $48 \mathrm{~h}$ after transfection. Renilla luciferase vector was used as an internal control. (D): Correlation between the ATAD2 mRNA level and the miR-520f was examined by qRT-PCR in 33 cases of gastric cancer tissues ( $p<0.001, \mathrm{r}^{2}=0.3215$, Pearson's correlation coefficient). The data presented are from three independent experiments. 
terparts, remarkable reduction of miR-520f was detected in all the 33 gastric carcinoma tissues (Figure 1B). These results show that the decreased miR-520f expression is a continual event in human gastric carcinoma cells and gastric carcinoma samples, suggesting that miR-520f may be involved in tumorigenesis.

MiR-520f inhibits growth of gastric carcinoma cells in vitro. To test the possible role of miR-520f in tumorigenesis, we identified whether miR-520f has influence on the growth of gastric carcinoma cells by 3-(4,5-dimethylthiazol-2-yl)-2, 5-diphenyltetrazolium bromide assay and 5-ethynyl-2'-deoxyuridine incorporation assay, respectively. MiR-520f inhibited the proliferation of MGC803 cells and anti-miR520f promoted the proliferation of GES-1 cells by MTT assay (Figure 2A-B). Moreover, EdU incorporation assay showed that miR-520f inhibited the proliferation of MGC803 cells and anti-miR520f promoted the proliferation of GES-1 cells (Figure 2C-D).

MiR-520f directly suppresses the expression of ATAD2 by targeting its 3 'UTR. To validate the mechanisms which miR-520f inhibits tumor growth, the candidate genes of miR$520 \mathrm{f}$ in human were screened from the TargetScan (http:// www.targetsca.org/). To determine whether ATAD2 is a direct target of miR-520f, we constructed pGL3-ATAD2-3'UTR-wt and pGL3-ATAD2-3'UTR-mut (Figure 3A). Over-expression of miR-520f remarkably inhibited the firefly luciferase activity of pGL3-ATAD2-3'UTR-wt but not that of pGL3-ATAD23'UTR-mut in MGC803 cells (Figure 3B). Furthermore, inhibition of endogenous miR-520f by anti-miR-520f led to enhanced firefly luciferase activity of the wild-type reporter but not that of the mutant one in GES-1 cells (Figure 3C).

\section{A

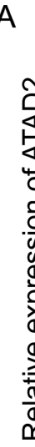

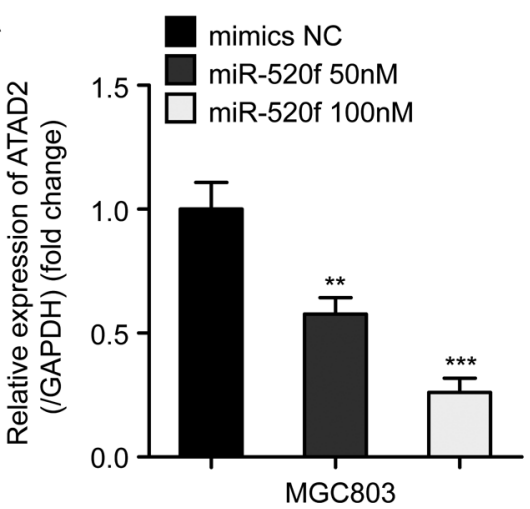

C

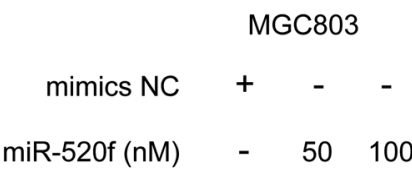

ATAD2

$\beta$-actin

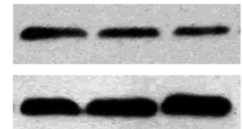

D

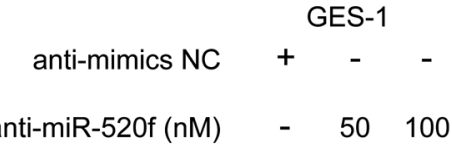

ATAD2

$\beta$-actin

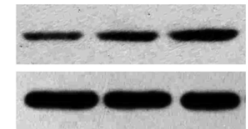

B

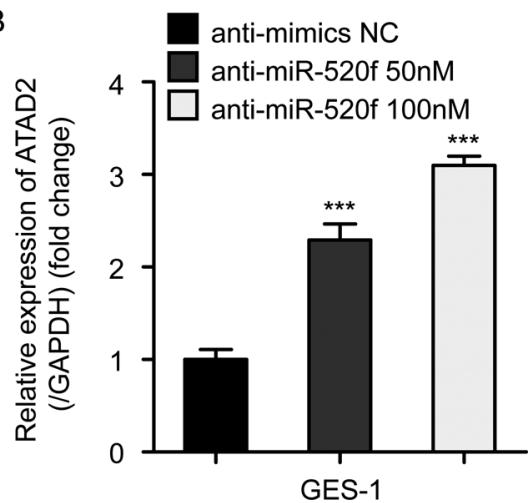

$E$

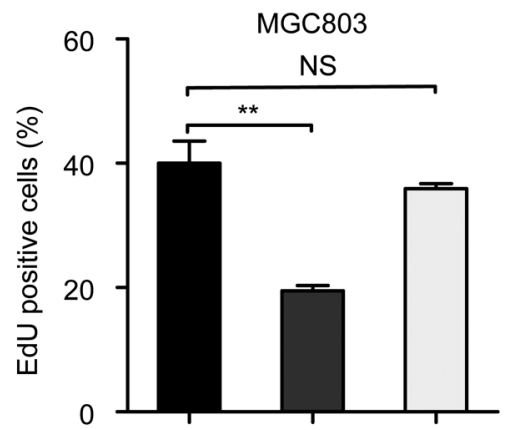

mimics NC +

$\mathrm{miR}-520 f$

pcDNA3-ATAD2

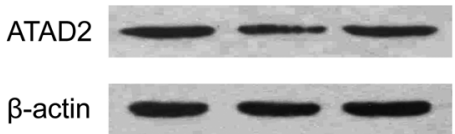

Figure 4. ATAD2 leads to miR-520f inhibited cell proliferation.

(A-B): Attenuation of ATAD2 expression by miR-520f. qRT-PCR analysis were used to detect the expression level of ATAD2 in MGC803 (GES-1) cells $48 \mathrm{~h}$ after transfection with $100 \mathrm{nM} \mathrm{NC}$ and 50 or $100 \mathrm{nM}$ miR-520f (100 nM mimics NC and 50 or $100 \mathrm{nM}$ anti-miR-520f). GAPDH was used as an internal control. Data are reported as mean \pm s.d. for three independent experiments $\left({ }^{\star *} \mathbf{P}<0.01 ;{ }^{* * \star} \mathbf{P}<0.001\right.$, Student's $t-$ test). (C-D) The expression level of ATAD2 in MGC803 and GES-1 cells treatment with miR-520f or anti-miR-520f were examined by western blot analysis. (E): EdU assay was performed to determine the growth of MGC803 cells co-transfected with miR-520f and pcDNA-ATAD2. The protein level of ATAD2 was measured by western blot. Data are reported as mean \pm s.d. for three independent experiments $\left({ }^{* *} \mathbf{P}<0.01\right.$, Student's $t$-test). 
Our data also demonstrated that the expression of ATAD2 was negatively correlated with that of miR-520f $(p<0.001$, $\mathrm{r}^{2}=0.3215$, Pearson's correlation coefficient).

ATAD2 leads to miR-520f inhibited cell proliferation. To test whether miR-520f expression has influence on endogenous ATAD2 expression, we transfected miR-520f or NC into MGC803 cells, the mRNA and protein level of ATAD2 was decreased (Figure 4A, C). At the same time, inhibit the expression level of miR-520f in GES-1 cells showed an increase of ATAD2 mRNA and protein level (Figure 4B, D). Moreover, the overexpression of ATAD2 partially reversed the inhibited proliferation mediated by miR-520f (Figure 4E). Thus, these data imply that miR-520f may weaken the expression of ATAD2 by directly targeting its 3'UTR.

\section{Discussion}

MiRNAs play an important role in biological processes, such as development, apoptosis, proliferation and differentiation, through imperfect pairing with target mRNAs of proteincoding genes, by the transcriptional or post-transcriptional regulation of their expression [15]. The initiation and progression of human cancer were regulated by a lot of MicroRNAs. However, the mechanism of miRNA involved in gastric carcinoma regulation remains to be further illustrated.

Gastric cancer migration, invasion and proliferation were affected by microRNA-25 directly targeting transducer of ERBB2, 1 [16]. The EBV-infected AGS gastric carcinoma cell lines also determined microRNA expression. Infected cells expressed the viral BamHI A rightward transcript (BART) miRNAs at high levels and had consistently decreased expression of a small fraction of cellular miRNAs with specific downregulation of tumor suppressor miRNAs [17]. It has been reported that miRNA-34a suppressed EGFR-signalingdependent MMP7 activation in gastric cancer [18]. Moreover, miRNA-296-5p increases proliferation in gastric cancer through repression of Caudal-related homeobox 1 [19]. In this study, we find miR-520f was a new tumor suppressor in gastric carcinoma tumorigenesis.

In hepatocellular carcinoma, ATAD2 as a poor prognostic marker after curative resection [20]. It has been reported that ATAD2 is up-regulated in gastric cancer and function as an independent poor prognostic biomarker. Overexpression and copy number alteration of ATAD2 are observed in many types of cancer, and these alterations are correlated with poor clinical outcome in breast, lung, and endometrial cancers [21]. Here, we find that ATAD2 was one target of miR-520f and contribute to gastric carcinoma tumorigenesis.

In this study, we reported the role of miR-520f in gastric carcinoma. Our finding suggests that miR-520f may be serving as a novel tumor-suppressor miRNA. MiR-520f blocks the growth of gastric carcinoma cells through targeting ATAD2. Our data provide new insight into the mechanism of gastric carcinoma. Additionally, miR-520f may serve as a possible therapeutic candidate in the treatment of gastric carcinoma.
Acknowledgements: We are indebted to the coworkers, whose names were not included in the author list, but who contributed to this work.

\section{References}

[1] VALADI H, EKSTROM K, BOSSIOS A, SJOSTRAND M, LEE JJ et al. Exosome-mediated transfer of mRNAs and microRNAs is a novel mechanism of genetic exchange between cells. Nat Cell Biol 2007; 9: 654-672. http://dx.doi.org/10.1038/ $\underline{\text { ncb1596 }}$

[2] HAINAUT P, PLYMOTH A. Targeting the hallmarks of cancer: towards a rational approach to next-generation cancer therapy. Curr Opin Oncol 2013; 25: 50-51. http://dx.doi. org/10.1097/CCO.0b013e32835b651e

[3] HANAHAN D, WEINBERG RA. Hallmarks of Cancer: The Next Generation. Cell 2011; 144: 646-674. http://dx.doi. org/10.1016/j.cell.2011.02.013

[4] ESTELLER M. Non-coding RNAs in human disease. Nature reviews Genetics 2011; 12: 861-874. http://dx.doi. org/10.1038/nrg3074

[5] CREW KD, NEUGUT AI. Epidemiology of gastric cancer. World J Gastroentero 2006; 12: 354-362. http://dx.doi. org/10.3748/wjg.v12.i3.354

[6] FEINBERG AP, TYCKO B. Timeline - The history of cancer epigenetics. Nat Rev Cancer 2004; 4: 143-153. http://dx.doi. org/10.1038/nrc1279

[7] GUO JM, MIAO Y, XIAO BX, HUAN R, JIANG Z et al. Differential expression of microRNA species in human gastric cancer versus non-tumorous tissues. Journal of gastroenterology and hepatology 2009; 24: 652-657. http://dx.doi. org/10.1111/j.1440-1746.2008.05666.x

[8] RAEDER MB, BIRKELAND E, TROVIK J, KRAKSTAD C, SHEHATA $S$ et al. Integrated Genomic Analysis of the 8q24 Amplification in Endometrial Cancers Identifies ATAD2 as Essential to MYC-Dependent Cancers. Plos One 2013; 8.

[9] BOUSSOUAR F, JAMSHIDIKIA M, MOROZUMI Y, ROUSSEAUX S, KHOCHBIN S. Malignant genome reprogramming by ATAD2. Bba-Gene Regul Mech 2013; 1829: 1010-1014. http://dx.doi.org/10.1016/j.bbagrm.2013.06.003

[10] CIRO M, PROSPERINI E, QUARTO M, GRAZINI U, WALFRIDSSON J et al. ATAD2 Is a Novel Cofactor for MYC, Overexpressed and Amplified in Aggressive Tumors. Cancer Res 2009; 69: 8491-8498. http://dx.doi.org/10.1158/00085472.CAN-09-2131

[11] WU G, LIU HY, HE H, WANG YW, LU XJ et al. miR-372 down-regulates the oncogene 3ATAD2 to influence hepatocellular carcinoma proliferation and metastasis. BMC Cancer $2014 ; 14$.

[12] CARON C, LESTRAT C, MARSAL S, ESCOFFIER E, CURTET $S$ et al. Functional characterization of ATAD2 as a new cancer/testis factor and a predictor of poor prognosis in breast and lung cancers. Oncogene 2010; 29: 5171-5181. http://dx.doi.org/10.1038/onc.2010.259

[13] RHO JK, CHOI YJ, KIM SY, KIM TW, CHOI EK et al. MET and AXL Inhibitor NPS-1034 Exerts Efficacy against Lung Cancer Cells Resistant to EGFR Kinase Inhibitors Because 
of MET or AXL Activation. Cancer Res 2014; 74: 253-262. http://dx.doi.org/10.1158/0008-5472.CAN-13-1103

[14] SACKSTEIN R, FUHLBRIGGE R. Western blot analysis of adhesive interactions under fluid shear conditions: the blot rolling assay. Methods in molecular biology 2015; 1312: 399-410. http://dx.doi.org/10.1007/978-1-4939-26947 39

[15] IORIO MV, CROCE CM. microRNA involvement in human cancer. Carcinogenesis 2012; 33: 1126-1133. http://dx.doi. org/10.1093/carcin/bgs140

[16] LI BS, ZUO QF, ZHAO YL, XIAO B, ZHUANG Y et al. MicroRNA-25 promotes gastric cancer migration, invasion and proliferation by directly targeting transducer of ERBB2, 1 and correlates with poor survival. Oncogene 2015; 34: 2556-2565. http://dx.doi.org/10.1038/onc.2014.214

[17] MARQUITZ AR, MATHUR A, CHUGH PE, DITTMER DP, RAAB-TRAUB N. Expression Profile of MicroRNAs in Epstein-Barr Virus-Infected AGS Gastric Carcinoma Cells.
J Virol 2014; 88: 1389-1393. http://dx.doi.org/10.1128/ IVI.02662-13

[18] LIU G, JIANG CS, LI DZ, WANG R, WANG W. MiRNA-34a inhibits EGFR-signaling-dependent MMP7 activation in gastric cancer. Tumor Biol 2014; 35: 9801-9806. http://dx.doi. org/10.1007/s13277-014-2273-6

[19] LI T, LU YY, ZHAO XD, GUO HQ, LIU CH et al. MicroRNA296-5p increases proliferation in gastric cancer through repression of Caudal-related homeobox 1. Oncogene 2014; 33: 783-793. http://dx.doi.org/10.1038/onc.2012.637

[20] HWANG HW, HA SY, BANG H, PARK CK. ATAD2 as a Poor Prognostic Marker for Hepatocellular Carcinoma after Curative Resection. Cancer Res Treat 2015; 47: 853-861. http:// dx.doi.org/10.4143/crt.2014.177

[21] TAKEDA T, MATSUMURA Y, NAGASAKI H, EGUCHI K, TAKATSUKA $M$ et al. Genomic alteration of bromodomain protein ATAD2 in cancer. Cancer Res 2014; 74: Abstract 2217 http://dx.doi.org/10.1158/1538-7445.AM2014-2217 